\title{
CortÁ: acercando cultura en cuarentena
}

\author{
Clara Pérez Pucci, ${ }^{1}$ Ignacio Bruné, ${ }^{2}$ Juan Andrés Frugoni, ${ }^{3}$ Leticia Lorier, ${ }^{4}$ \\ Magela Rodríguez, ${ }^{5}$ Marcela Tancredi, ${ }^{6}$ María Lilian González, ${ }^{7}$ \\ Néstor Bermúdez, ${ }^{8}$ Patricia Sedes ${ }^{9}$
}

DOI: https://doi.org/10.37125/ISR.7.1.10

\section{Resumen}

Esta reseña presenta el proyecto «CortÁ: cortometrajes accesibles. Recepción y adecuación de componentes de SPS, LS y AD en contenidos audiovisuales», que se llevó adelante en el contexto de la primera edición del Espacio de Formación Integral Comunicación y Accesibilidad de la Facultad de Información y Comunicación y la Facultad de Humanidades y Ciencias de la Educación de la Universidad de la República. El trabajo enfatiza en los aciertos, posibilidades y desafíos de esta propuesta integral e interdisciplinaria. En primera instancia, se desarrollan los antecedentes que lo posibilitan y justifican; luego, se presentan el recorrido y las tensiones entre el diseño y ejecución del EFI en el marco de la emergencia social y sanitaria de covid-19. El artículo finaliza con la presentación de la evaluación realizada por los docentes y estudiantes participantes y las proyecciones de trabajo asociadas.

Palabras clave: Derechos humanos, discapacidad, accesibilidad.

\section{Fundamentación y antecedentes}

El proyecto de extensión «CortÁ: cortometrajes accesibles. Recepción y adecuación de componentes de SPS, LS y $\mathrm{AD}^{10}$ en contenidos audiovisuales» resultó aprobado en la Convocatoria a Proyectos de Extensión relativos a Derechos Humanos 2020 de la Comisión Sectorial de Extensión y Actividades en el Medio (CSEAM). El proyecto

1 Licenciatura en Lingüística, Facultad de Humanidades y Ciencias de la Educación, Universidad de la República (FHCE, Udelar). claraperezpucci@hotmail.com.

2 Licenciatura en Comunicación, Facultad de Información y Comunicación (FIC), Udelar. ignaciobrune@gmail.com.

3 Tecnicatura Universitaria en Interpretación y Traducción de Lengua de Señas Uruguaya (TUILSU), FHCE, Udelar.

FIC, Udelar.

Licenciatura en Comunicación, FIC, Udelar.

FHCE, Udelar.

Servicio Central de Extensión y Actividades en el Medio, Udelar.

8 Licenciatura en Lingüística, FHCE, Udelar.

9 TUILSU, FHCE, Udelar.

10 SPs: 'subtitulado para sordos'; Ls: 'lengua de señas'; AD: 'audiodescripción'. 
original incluía el diseño de un ciclo de exhibición, estudio y sensibilización con cortometrajes uruguayos que incorporaran componentes de accesibilidad audiovisual: lengua de señas, subtitulado descriptivo (SD) y audiodescripción. Para ello, se generaron las herramientas accesibles de cortometrajes seleccionados con alternativas de SD, LS y AD, ya que el objetivo era focalizar en la recepción de tales componentes con el fin de indagar cuáles eran las formas más adecuadas para los destinatarios uruguayos. Esta planificación original tuvo que ser revisada debido a la emergencia social y sanitaria atravesada por nuestro país. Sin embargo, la fundamentación del proyecto de extensión, en lugar de revisarse, tomó mayor visibilidad. La emergencia social y sanitaria recrudeció las barreras que impiden a colectivos de personas en distintas condiciones de vulnerabilidad el ejercicio de sus derechos en condiciones de igualdad y no discriminación (personas en situación de discapacidad, sordos, adultos mayores, migrantes, entre otros).

Existen diversos marcos normativos, tanto nacionales como internacionales, que reconocen y aseguran derechos a estos colectivos de personas en condiciones de vulnerabilidad. Uno de estos es la Convención Internacional de los Derechos de las Personas con Discapacidad (Organización de las Naciones Unidas [ONU], 2006), el primer tratado de derechos humanos del siglo xxi, al cual Uruguay adhirió mediante la Ley n. $^{0} 18.418$ en 2008 (Uruguay, 2008b). Al materializar este tratado internacional de protección de derechos humanos en la legislación nacional, nuestro país adquirió compromisos, es decir, el Estado se ve obligado a proteger y garantizar el pleno goce y ejercicio de los derechos que se encuentran en dicho tratado, que impactan en la cotidianeidad y calidad de vida de las personas en situación de discapacidad. La convención refiere en su artículo 9 a la accesibilidad, en su artículo 21 a la libertad de expresión y en el artículo 30 al ocio y esparcimiento. Se estipula que el acceso a la cultura es un derecho y los productos audiovisuales deben ser presentados en diversos formatos en aras de garantizarlo. De esta misma forma, en 2001, se promulgó la Ley n. ${ }^{\circ} 17.378$ (Uruguay, 2001), que en su artículo primero reconoce la lengua de señas uruguaya (LSU) como lengua natural de las personas sordas y sus comunidades, y se plantea como objetivo eliminar las barreras comunicacionales existentes para estas comunidades.

En 2008 se aseguraron derechos de las personas migrantes: la Ley n. ${ }^{\circ} 18.250$ (Uruguay, 2008a) en su primer capítulo establece la igualdad de derecho con los nacionales, y en el tercero se reconoce el derecho al acceso a la información y la integración sociocultural de las personas migrantes. La Ley n. ${ }^{\circ} 19.430$ (Uruguay, 2016) reconoce y adhiere al tratado de la Convención Interamericana sobre la Protección de los Derechos Humanos de las Personas Mayores, que en su artículo 14 reconoce el derecho a la libertad de expresión, de opinión y al acceso a la información. Es atendiendo a este marco normativo que este proyecto pretende contribuir al trabajo en conjunto con las personas que, sin contar con componentes de accesibilidad 
comunicacional, no tienen garantizado el goce de derechos como el acceso a la cultura y al ocio.

El Espacio de Formación Integral (EFI) en el que se enmarca este proyecto de extensión aborda la accesibilidad desde una perspectiva de derechos humanos para dar respuesta a las barreras que una porción de la población (personas en situación de discapacidad, personas sordas, adultos mayores, migrantes, entre otros) encuentra para acceder a la información, la comunicación y la cultura.

El movimiento social por los derechos de las personas en situación de discapacidad tiene la máxima nada sobre las personas en situación de discapacidad sin las personas en situación de discapacidad. Este lema sintetiza la lucha de un colectivo que históricamente ha tenido un rol periférico en la toma de decisiones, de acciones, de desarrollos que tienen un impacto directo en su calidad de vida. Este lema responsabiliza a quienes se embarcan en la ejecución de proyectos asociados a la temática de incluir a los colectivos en todas las fases de acción, con una participación activa y reconociendo sus saberes. Es en este sentido que en este proyecto de extensión participaron la Asociación de Sordos del Uruguay (ASUR) y la Unión Nacional de Ciegos del Uruguay (UNCU), dos de las organizaciones de la sociedad civil más antiguas del Uruguay que luchan por la reivindicación y el reconocimiento de los derechos de los sordos y de las personas en situación de discapacidad visual. En el caso de AsUR, ha venido conquistando derechos de acceso a la cultura con la inclusión en la educación de la LSU desde 1989. Restan aún los espacios de recreación y entretenimiento y la inclusión progresiva de la interpretación en LSU en la televisión de aire uruguaya a través de la Ley n. ${ }^{\circ} 19.307$ (Uruguay, 2014), conocida como Ley de Medios, promulgada en el 2014 y regularizada por el poder ejecutivo en el 2019. La Red MATE de UNCU ha realizado ya, en tres ediciones, un ciclo de cine accesible coorganizado por Okurelo Cine, la Universidad Católica del Uruguay y el Centro Cultural España. La edición de 2020 también contó con la coorganización de la Facultad de Información y Comunicación (FIC) como parte del plan para la etapa de exhibición de este proyecto.

Mención aparte merece el hecho de que la Universidad de la República (Udelar) no toma el tema de la accesibilidad audiovisual (AAV) como parte de su currícula; es allí donde la propuesta del proyecto y la continuidad del EFI se hace relevante. La Udelar no tenía hasta el momento la temática en sus currículos, dado que quienes egresan no consideran la accesibilidad comunicacional como una posible fuente de trabajo o como una tarea que les puede ser encomendada. A partir de estos procesos es que se identificó, en conjunto con las instituciones, la necesidad de trabajar sobre la temática AAV.

Con respecto a los antecedentes específicos del espacio de formación, se destacan el EFI Literatura en LSU —-dedicado a la adecuación de textos literarios clásicos en español a la LS, en el que se produjo textualidad en LSU con subtítulos para que fueran accesibles para la comunidad oyente- y el EFI Subtitulado, con dos ediciones (2017 y 2018), enfocado en el subtitulado para sordos. En este último participaron 
estudiantes sordos y oyentes, por lo que también se abordó de forma introductoria el subtitulado de videograbaciones en LSU, cuyo público objetivo es la comunidad oyente. En el marco de este EFI, se tomó contacto con jóvenes sordos estudiantes del Liceo n. ${ }^{\circ} 32$ de Montevideo para indagar sobre preferencias y adecuación de la elaboración de los subtítulos en varios de los parámetros usados en el sPs. Además, la Tecnicatura en Interpretación y Traducción Lengua de Señas Uruguaya-Español (TUILSU) de la Facultad de Humanidades y Ciencias de la Educación (FHCE), a través de su programa de extensión e investigación TRELSU (Textualidad Registrada en Lengua de Señas Uruguaya), trabaja sobre la lingüística de la LsU, la producción de videograbaciones de LSU como escritura, la traducción del español escrito a la LSU videograbada y el estudio sobre el subtitulado descriptivo.

Finalmente, desde 2016, la FIC viene trabajando en el área de traducción audiovisual, vinculando el campo de la comunicación, las ciencias del lenguaje y los estudios de traducción. En ese marco se incluye el Laboratorio de Traducción Audiovisual como oferta de Educación Permanente, del que participaron docentes de la Udelar y de universidades extranjeras y que ya tuvo su tercera edición en el 2019; en 2020 se ofreció el curso de Educación Permanente Accesibilidad Audiovisual: Módulo Subtitulado. Desde 2017, la temática de la accesibilidad se incorpora con módulos dedicados específicamente al SD, la Ls y la AD. Además, en 2019 se organizó el Ciclo de Accesibilidad Audiovisual, apoyado por el Espacio Interdisciplinario, en el que se desarrollaron diferentes actividades de formación, divulgación y experimentación en AAv. En él participaron especialistas y académicos de Uruguay, Argentina, Brasil y España y representantes del sector público y privado, así como organizaciones civiles.

\section{Presentación del proyecto CortÁ}

El proyecto «CortÁ: cortometrajes accesibles. Recepción y adecuación de componentes de SPS, LS y AD en contenidos audiovisuales» se propuso desde su diseño una metodología de investigación-acción (Lewin, 1992) que integrara a la población destinataria como actores protagonistas en todo el proceso. Una de sus líneas transversales es la indagación sobre diferentes posibilidades en las formas en que se hace la audiodescripción, la inserción de los subtítulos y la lengua de señas uruguaya. Mediante distintas dinámicas de exploración, se propuso identificar qué formas resultan mejores para los destinatarios uruguayos y por qué el espectador puede dar sentido completo y no fragmentado a la obra. En este sentido, CortÁ tiene una dimensión de investigación del tipo exploratoria. Se propuso un abordaje enfocado en la recepción de los contenidos accesibles y que se encuadra metodológicamente dentro del tipo de estudios realizados en el campo de la traducción audiovisual.

El objetivo general propuesto fue generar un espacio de promoción de derechos humanos, en el que se fomentara el derecho a la comunicación, la información y el acceso a la cultura por medio de la accesibilidad audiovisual. Los objetivos específicos 
fueron: 1) generar conocimiento interdisciplinario sobre comunicación y accesibilidad; 2) hacer un estudio de recepción que permita avanzar en estudios de cuáles son las formas más adecuadas de los componentes para los destinatarios uruguayos, y 3) generar cortos audiovisuales con componentes de accesibilidad: lengua de señas uruguaya, subtitulado descriptivo y audiodescripción. Además, se propuso hacer un ciclo de exhibición que permita que tanto los usuarios destinatarios y sus comunidades puedan disfrutar de una experiencia cinematográfica accesible.

El proyecto se diseñó en cuatro grandes campos de desarrollo: formación (julio-agosto 2020), producción (setiembre 2020-febrero 2021), exhibición (octubre-noviembre 2020) y estudio de recepción (diciembre 2020-abril 2021). Las particularidades del año influenciaron en que el cronograma previsto haya tenido que sufrir ajustes y modificaciones, que se presentarán más adelante.

El bloque de formación estuvo compuesto por cuatro módulos temáticos: accesibilidad y audiovisual, audiodescripción, subtitulado y lenguas de señas en medios audiovisuales. En el primer módulo se incluyó una presentación específica sobre el enfoque de derechos humanos asociados a la discapacidad y cómo este se relaciona con una noción más amplia sobre el abordaje del concepto de accesibilidad. Asimismo, fueron trabajados aspectos propios del lenguaje audiovisual y su pertinencia con el eje principal: la accesibilidad. Los siguientes módulos fueron enfocados en las especificidades de cada herramienta:

- audiodescripción, entendida como la locución de información significativa de una imagen, con el fin de describirla dentro de una pieza audiovisual;

- subtitulado descriptivo, entendido como una combinación de texto y gráficos en pantalla en representación de los discursos orales, el contenido verbal y no verbal, la información suprasegmental y los efectos sonoros, que pueden ser tanto intralingüísticos como interlingüísticos —en suma, una simplificación textual o efectos gráficos con el fin de enriquecer la comprensión-, y

- lengua de señas, en la que se empleó la lengua de señas uruguaya, lengua natural de la comunidad sorda del Uruguay que, como otras lenguas de señas, se caracteriza por ser visogestual y percibida con la mirada. En el audiovisual, como los subtítulos, es un elemento más de la imagen.

Una vez culminada la etapa de formación (julio-agosto), se dividió a los estudiantes por herramienta (esto es, un subgrupo se dedicó a la AD, otro al SD, etcétera); cada grupo tenía un docente referente. Los distintos subgrupos de trabajo fueron interdisciplinarios (en todos los subgrupos participaron estudiantes de ambas facultades). La conformación, a su vez, tuvo en cuenta que el campo de producción estuviera en sintonía con las formaciones académicas de los estudiantes y sus preferencias particulares a la hora de trabajar con los cortometrajes. 
En la etapa de producción (setiembre-febrero), se trabajó con tres cortometrajes uruguayos. Tanto la selección de los audiovisuales como la cesión de derechos fue posible gracias al trabajo en conjunto con Montevideo Audiovisual, que integra el departamento de Cultura de la Intendencia de Montevideo. Estos fueron tres audiovisuales de ficción uruguayos: La peste, cortometraje de los géneros drama y horror dirigido por Guillermo Carbonell y producido por Murdoc en 2017; La esperanza, cortometraje del género drama del director Martin Estizarreguy y datado en 2015, y el capítulo «Lunas con dormilones», de Artistonautas, una serie animada infantil dirigida por Juan Carve y producida por Chucho.tv en 2015. En esta etapa, se subdividieron nuevamente grupos de estudiantes y fue por cortometraje a intervenir. Los estudiantes, con el acompañamiento de los docentes referentes, se encargaron del guionado de la AD, el diseño de la Ls y el SD respectivamente, es decir, de delimitar los aspectos creativos de cada herramienta en sus dos versiones: una tradicional, que se ajustaba a recomendaciones o estándares existentes, y otra creativa, con una apuesta de la integración de la herramientas de accesibilidad en mayor consonancia con la propuesta estética y narrativa del film y que a su vez tenía por objetivo resultar una mejor experiencia de recepción para los usuarios.

Finalmente, se reorganizaron los diferentes subgrupos según las disciplinas y tareas a ejecutar en la fase final de producción del proyecto. Dependiendo de la formación específica de cada estudiante, le fue asignado un nuevo grupo de trabajo. Estos grupos fueron los encargados del montaje, corrección de estilo, locución, cámara, edición de sonido, traducción español-LSU, entre otros.

Cada subgrupo tuvo docentes orientadores de conformación interdisciplinaria. Se diagramó la fase de producción como continuación de la formación y con una fuerte apuesta al desarrollo de exploración creativa. Las tareas de edición y montaje fueron llevadas a cabo por estudiantes de la Licenciatura en Comunicación de la FIC; la corrección de estilo de la locución de la AD y los SD fue asignada a estudiantes de la Licenciatura en Lingüística y de la Tecnicatura Universitaria en Corrección de Estilo de la FHCE, y la traducción de español a LSU fue trabajada por estudiantes de la TUILSU.

Siguiendo la máxima referida antes, el proyecto CortÁ fue planificado con la intención de generar instancias de testeo de las herramientas de accesibilidad con las organizaciones sociales y sus integrantes en representación de los usuarios finales. Una vez adaptados los cortometrajes, un primer borrador de la locución de la $\mathrm{AD}$ fue testeado por unCU. Dadas las restricciones sanitarias, en esa instancia participó un estudiante por cortometraje. Estos fueron presentados a un grupo compuesto por tres mujeres adultas y dos jóvenes. Luego del intercambio, en el cual los sujetos dieron sus comentarios sobre el trabajo realizado y sus posibles mejoras, los estudiantes recogieron los datos surgidos en la validación e hicieron los ajustes pertinentes para la construcción de una nueva grabación de la AD. En lo que respecta al testeo de la LSU y el SD, no se logró la coordinación de una instancia de testeo con 
ASUR; sin embargo, hubo participación activa de personas sordas en todo el proceso del EFI, ya que contaba con estudiantes y docentes sordos de la TUILsu. Esto permitió desarrollar valederos conocimientos y sortear desafíos no solo a nivel formativo, sino también experiencial, lo cual fue clave a la hora de trabajar con los cortometrajes.

A raíz de la situación sanitaria, la cuarta etapa, en que se preveía la exhibición en liceos y escuelas de los cortometrajes adaptados en el marco del EFI, tuvo que ser ajustada. Es así que fue incorporado al EFI para participar del Ciclo de Cine Accesible en su edición 2020, organizado por la UNCU y coorganizado por otras organizaciones, entre ellas la FIC. En ese marco, estudiantes del EFI desarrollaron gran parte de los materiales de difusión, actuaron como orientadores en las salas de exhibición y colaboraron con la implementación de la versión online del ciclo. El cortometraje La peste formó parte de la muestra con las distintas herramientas de accesibilidad en sus versiones creativas, tanto en la instancia presencial en la sala Zitarrosa en noviembre de 2020 como la versión virtual en la web ciclocineaccesible.fic.edu.uy. Cabe destacar que a la sala Zitarrosa concurrió el director La peste y luego envió una evaluación muy positiva respecto a cómo fue adaptada su obra.

Siguiendo con la planificación y objetivos del proyecto, se está ejecutando, a la hora de redactar esta reseña, un estudio de recepción exploratorio sobre las preferencias de la audiencia uruguaya. Como objetivo general, este estudio se plantea aproximarse a las preferencias de consumo audiovisual relacionado con las herramientas de accesibilidad (SD-AD-LSU) por parte de sus respectivos usuarios (personas sordas o de escucha reducida y personas ciegas o con pérdida de visión). Como objetivos específicos, este estudio se plantea, por un lado, explorar las preferencias, el disfrute y la sensación de inmersión de los usuarios ciegos y con baja visión sobre dos variantes diferenciadas de interpretación de la voz en AD. Por otro lado, explorar las preferencias, disfrute y sensación de inmersión de los usuarios sordos y con escucha reducida sobre dos variantes de la interpretación en LSU y el SD. Vale remarcar que este estudio tiene la intención de ser un estudio de corte exploratorio: más que conclusiones, se propone llegar a plantearse nuevas preguntas a partir de los resultados obtenidos.

\section{Aciertos y desafíos: particularidades pandémicas}

Este proyecto se enmarca dentro de la actual emergencia sanitaria de covid-19, la cual causó que actividades pensadas para la presencialidad pasaran a ser estrictamente virtuales - como lo fue la etapa de formación - o con contados encuentros presenciales bajo estrictos protocolos, como los talleres de voz y las grabaciones de la AD y la LS. Sin embargo, el buen manejo de las herramientas virtuales (plataforma EVA, sala de videoconferencia, trabajos virtuales) por parte del cuerpo docente, sumado a un acompañamiento y monitoreo constante, permitieron que el proyecto de extensión fuera exitoso y cómodo de ejecutarse. 


\section{Aspectos creativos}

En lo que refiere a los cortometrajes trabajados durante el proyecto de extensión, se decidió tomar dos enfoques por herramienta de accesibilidad. Un enfoque tradicional, en el que se usaron guías y estándares, como la de la RECAM (Bahiense, Mauch, Ferreira y Santiago, 2017) o la de la Universidad de Varsovia (Szczygielska, 2020), como base para la aplicación de las herramientas, y otro enfoque integrado a la obra, para el que se utilizaron elementos creativos para experimentar con nuevos formatos y procedimientos. Así, se realizó una audiodescripción con aspectos performáticos y entonacionales marcados, un subtitulado creativo e integrado con la intención de seguir la línea estética de cada cortometraje, experimentando con el posicionamiento, efectos gráficos, fondo, tipografía, entre otros, y la traducción a la lengua de señas uruguaya, con la que se experimentó en los aspectos relacionados con el posicionamiento, el fondo, el vestuario y el tamaño del señante para ir acorde a la realidad del audiovisual. Además, los señantes en pantalla fueron personas sordas: esto fue asumido como una postura en el entendido de que la LSU es la lengua natural y patrimonio lingüístico de la comunidad sorda del Uruguay.

\section{Desafíos docentes}

Desde el equipo docente, resulta fundamental transmitir los distintos niveles de complejidad que generó el desafío de planificar y desarrollar este EFI en formato virtual. En primera instancia, se hace necesario reconocer que los docentes que conforman el equipo provienen de distintos espacios de la Udelar e integran socios externos, lo cual conlleva numerosas coordinaciones y articulaciones, además de acompasar ritmos y metodologías de trabajo distintas. Asimismo, resulta oportuno destacar algunos aprendizajes que implicó esta complejidad: el rediseño y trabajo con lo acaecido durante el proyecto de extensión, los ajustes propios del encuentro virtual y la postergación de algunas planificaciones. Dentro de las postergaciones, se incluyen las actividades de proyección que se preveían tanto en espacios públicos como en instituciones educativas.

Al ser la primera edición del proyecto CortÁ, también fue para el equipo docente un ejercicio de conocimiento en sí mismo, ya que para muchos docentes es la primera experiencia de trabajo conjunto. Se reconoce que el motor estuvo en la motivación y convicción de que espacios de este estilo son necesarios. También resultó necesario el desarrollo de conocimiento en conjunto sobre los cruces temáticos que confluyeron en el proyecto de extensión.

Es fundamental reconocer el compromiso y dedicación de cada una y cada uno de los estudiantes que participaron en esta propuesta de CortÁ; desde el equipo docente existe plena consciencia de que 2020 fue un año particularmente difícil y se destaca el entusiasmo y la responsabilidad presentes en respuesta a cada una de las tareas propuestas. 
Por último, y considerando que este desafío es medular en CortÁ, se destaca la planificación e incorporación de criterios de accesibilidad en todos los espacios asociados: los materiales, las tareas propuestas, las clases sincrónicas y asincrónicas. Esta definición de que la propuesta formativa sea accesible en sí misma permitió vivenciar la complejidad y llegar a la conclusión de que es posible generar propuestas accesibles desde la Universidad de la República.

\section{Círculo academia-sociedad}

La accesibilidad comunicacional en contextos culturales es incipiente en la región. Dado que la incorporación de componentes de accesibilidad es reciente, también lo es el desarrollo de estas herramientas. Como sucede con el doblaje y el subtitulado, las características del territorio y el contexto de cada audiencia generan preferencias sobre cómo deben ser desarrollados. Se exploraron, entonces, estas preferencias de la audiencia receptora y usuaria. Durante el proceso de trabajo siempre estuvo presente el vínculo con la sociedad civil, específicamente con UNCU y ASUR. Primero, a través de diferentes encuentros virtuales, en los que expusieron su trabajo social y organizativo; luego, a través de los testeos de las herramientas de accesibilidad empleadas en los cortometrajes, y, por último, como espectadores de La peste en la sala Zitarrosa. Esto permitió que el proyecto de extensión no solo completara su cometido, sino que también formara un círculo de trabajo interdisciplinario entre facultades y la sociedad civil.

En lo que refiere específicamente a los estudiantes sordos, esta experiencia mostró las dificultades de la comunidad sorda para acceder tanto a los contenidos culturales como a la información en general. En este EFI se logró sobrellevar quizás la más grande de todas: la barrera lingüística existente entre individuos oralizados e individuos que, con mayor o menor conocimiento del español, tienen la LSU como primera lengua. El trabajo, coordinación y adaptación a las vicisitudes de la pandemia y la virtualidad lograron que varios estudiantes sordos pudieran participar con la inclusión de intérpretes de LSU en el equipo del EFI, cuyo conocimiento, junto al trabajo conjunto del cuerpo docente y estudiantil, fue lo que permitió una mayor conexión con la sociedad civil y dio insumos metodológicos, pedagógicos y didácticos para hacer accesible el conocimiento y la información, además de desafíos en contextos complejos como el suscitado durante el EFI.

\section{Evaluación de los integrantes de CortÁ en la actividad de cierre}

En la evaluación final del curso se obtuvieron un total de 16 respuestas, ilustradas en el Gráfico 1. En el apartado «El equipo docente transmitió claridad conceptual en los temas propuestos», todos los participantes respondieron de forma afirmativa: la opción «totalmente de acuerdo» fue la más votada, con trece respuestas. 
En lo referente al apartado «La construcción con los colectivos involucrados fue una instancia de aprendizaje novedosa», doce participantes votaron «totalmente de acuerdo» y cuatro respondieron «de acuerdo». En el apartado «La integración sub-grupal (por herramienta, por corto, por tarea) fue una metodología adecuada para el desarrollo del curso», diez de las respuestas emitidas entraron en la opción «totalmente de acuerdo» y seis respuestas en «de acuerdo».

\section{Figura 1. \\ Evaluación de los integrantes del EFI en la actividad de cierre.}

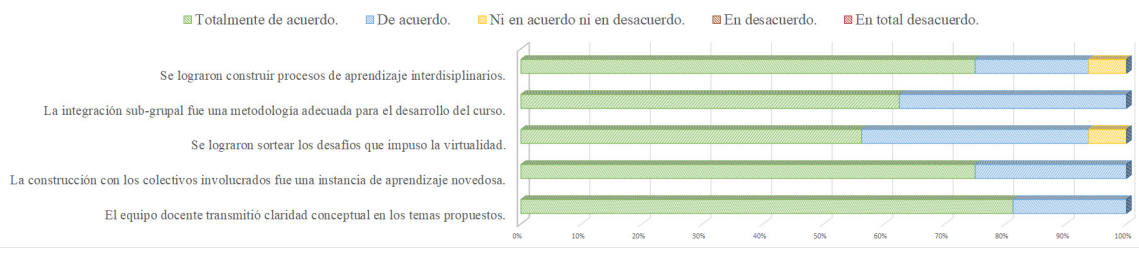

Fuente: elaboración propia.

En la opción «Se lograron sortear los desafíos que impuso la virtualidad», nueve integrantes respondieron "totalmente de acuerdo», seis respondieron «de acuerdo" y uno «ni en acuerdo ni en desacuerdo», y en la opción «Se lograron construir procesos de aprendizaje interdisciplinarios», doce integrantes del EFI contestaron «totalmente de acuerdo», tres respondieron «de acuerdo» $\mathrm{y}$ uno «ni en acuerdo ni en desacuerdo».

Respecto a las proyecciones, resulta importante mencionar que el EFI en el que se contextualiza CortÁ fue uno de los antecedentes principales para la postulación al llamado a Núcleos Interdisciplinarios promovido por el Espacio Interdisciplinario de la Udelar. La propuesta presentada fue el «Núcleo Interdisciplinario Comunicación y Accesibilidad», que resultó financiado y se ejecutará en el período 2021-2023. Se planifican nuevas ediciones de este proyecto de extensión en el marco del Núcleo, sumando la participación de otros servicios universitarios como Facultad de Psicología y Facultad de Arquitectura, Diseño y Urbanismo. Además, en el EFI se prevé la exploración entorno a una nueva herramienta de accesibilidad que es la lectura fácil y su articulación con los sistemas aumentativos y alternativos de comunicación. Desde la perspectiva estudiantil, tanto el EFI como el proyecto Cort Á son de suma importancia académica y de compromiso cívico y democrático. Los elementos de la interdisciplinariedad y del trabajo en conjunto, el uso óptimo de las diversas plataformas virtuales y todo el proceso de creación, testeo y re-creación fueron clave para poder llevar a cabo lo requerido por el cuerpo docente. Si bien el contexto en el que se llevó adelante el EFI fue complejo de gestionar por lo impredecible de la emergencia sanitaria, se logró no solo cumplir con los objetivos, sino inspirar a muchos y muchas estudiantes a continuar con esta línea tanto en iniciativas de investigación y extensión, así como de desarrollo profesional, algo que es de suma importancia para la expansión de los saberes creados junto con la sociedad civil. 
En suma, CortÁ se propuso, desde su diseño, la articulación de funciones universitarias. La extensión estuvo presente en la propia elaboración del proyecto, ya que incluyó, como se ha mencionado, talleres de validación y cocreación durante todo el proceso, así como la realización de un ciclo de exhibición de los cortos con componentes de accesibilidad. La enseñanza transversaliza toda la ejecución, desde la oferta de distintas actividades de formación sincrónica y asincrónica hasta la elaboración de las diferentes herramientas para formar a los estudiantes participantes. La producción de conocimiento prevista estaba asociada al estudio exploratorio de recepción. Este componente es el que se vio más afectado por el contexto de pandemia, ya que se debieron postergar varias actividades hasta recuperar espacios de presencialidad y encuentro. La valoración general del proyecto es de una propuesta novedosa y necesaria para el ejercicio de derechos de algunos colectivos.

Reafirmamos el compromiso universitario de aportar a la construcción de una sociedad más inclusiva. Entendemos y promovemos la extensión como una herramienta para transversalizar en la práctica universitaria la premisa del movimiento social de personas en situación de discapacidad y que aplica a otros colectivos: nada de nosotros sin nosotros.

\section{Referencias}

Bahiense, S., Mauch, C., Ferreira S., y Santiago, V. (Orgs.) (2017). Guía para producciones audiovisuales accesibles. Montevideo: Intendencia de Montevideo-RECAM.

LEWIN, K. (1992). La investigación-acción y los problemas de las minorías. En M. C. SALAZAR (Ed.), La investigación-acción participativa. Inicios y desarrollos (pp. 13-26). Madrid: Editorial Popular-OEIQuinto Centenario.

Organización de las Naciones Unidas (ONU) (2006, mayo 3). Convención sobre los derechos de las personas con discapacidad. Recuperado de https://www.un.org/esa/socdev/enable/documents/ tccconvs.pdf.

SzCZYGIELSKA, M. (2020). Guidelines on the provision of sign language interpreting in the media in times of crisis. Varsovia: Universidad de Varsovia. Recuperado de https://avt.ils.uw.edu.pl/files/2020/03/ Guidelines-on-the-provision-of-sign-language-interpreting-in-the-media-in-times-of-crisis.pdf.

Uruguay (2001, julio 25). Ley n. ${ }^{\circ}$ 17.378: Personas discapacitadas. Lengua de Señas Uruguaya. Recuperado de https://www.impo.com.uy/bases/leyes/17378-2001.

Uruguay (2008a, enero 6). Ley n. ${ }^{\circ}$ 18.250: Ley de Migraciones. Recuperado de https://www.impo.com.uy/ bases/leyes/18250-2008.

URUGUaY (2008b, diciembre 4). Ley n. ${ }^{\circ}$ 18.418: Convención de Naciones Unidas sobre los derechos de las personas con discapacidad. Recuperado de https://www.impo.com.uy/bases/leyes/18418-2008.

Uruguay (2014, diciembre 29). Ley n. ${ }^{\circ}$ 19.307: Ley de Medios. Regulación de la prestación de servicios de radio, televisión y otros servicios de comunicación audiovisual. Recuperado de https://www. impo.com.uy/bases/leyes/19307-2014.

URUguay (2016, setiembre 8). Ley n. ${ }^{\circ}$ 19.430: Aprobación de la Convención Interamericana Sobre la Protección de los Derechos Humanos de las Personas Mayores. Recuperado de https://www.impo. com.uy/bases/leyes/19430-2016. 